\title{
Erratum
}

\section{Towards a better understanding of adolescent obsessive-compulsive personality traits and obsessive-compulsive symptoms from growth trajectories of perfectionism - ERRATUM}

\author{
Selim Sametoğlu, Jaap J.A. Denissen, Barbara De Clercq and Elien De Caluwé
}

doi: https://doi.org/10.1017/S0954579421000195. Published online by Cambridge University Press, 12 April 2021.

Keywords: child and adolescent perfectionism, latent growth curve modeling, obsessive-compulsive disorder, obsessive-compulsive personality disorder

In Sametoğlu et al. 2021, as a result of a production error there is a mistake in table 1 , column 1 , row 12. The text should read "Order/Clean/Perfect Domain." The correct table 1 follows:

Table 1. Overview of standardized regression coefficients when regressing obsessive-compulsive personality disorder (OCPD) and obsessive-compulsive disorder (OCD) variables on intercept values from latent growth model for perfectionism

\begin{tabular}{|c|c|c|c|}
\hline & $\begin{array}{c}1 \text { st } \\
\text { model }\end{array}$ & $\begin{array}{l}\text { 2nd } \\
\text { model }\end{array}$ & $\begin{array}{c}3 \mathrm{rd} \\
\text { mode }\end{array}$ \\
\hline OCPD mean & $.23^{\star \star \star}$ & & \\
\hline Perseveration & & $.14^{*}$ & $.14^{\star}$ \\
\hline Rigid Perfectionism & & $.39^{\star \star \star}$ & $.39^{\star \star \star}$ \\
\hline Intimacy Avoidance & & .07 & .07 \\
\hline Restricted Affectivity & & .03 & .03 \\
\hline OCD mean & $.23^{\star \star \star}$ & & \\
\hline Obsessions Domain & & $.17^{\star \star}$ & \\
\hline Aggression & & & .11 \\
\hline Guilt & & & $.14^{\star}$ \\
\hline Physical Appearance & & & .10 \\
\hline Somatization & & & $.20^{\star \star}$ \\
\hline
\end{tabular}

Table 1. (Continued.)

\begin{tabular}{|c|c|c|c|}
\hline & $\begin{array}{c}1 \text { st } \\
\text { model }\end{array}$ & $\begin{array}{l}\text { 2nd } \\
\text { model }\end{array}$ & $\begin{array}{c}3 \text { rd } \\
\text { model }\end{array}$ \\
\hline $\begin{array}{l}\text { Order/Clean/Perfect } \\
\text { Domain }\end{array}$ & & $.34^{\star \star \star}$ & \\
\hline Cleanliness & & & $.27^{\star \star \star}$ \\
\hline Orderliness & & & $.26^{\star \star \star}$ \\
\hline Perfectionism & & & $.36^{\star \star \star}$ \\
\hline Compulsions Domain & & .05 & \\
\hline Hoarding & & & .01 \\
\hline Magic games & & & .05 \\
\hline Repeating & & & .07 \\
\hline
\end{tabular}

${ }^{\star} p<.05 .{ }^{* \star} p<.01 .{ }^{* \star \star} p<.001$.

\section{Reference}

Sametoğlu, S., Denissen, J. J. A., De Clercq, B., \& De Caluwé, E. (2021). Towards a better understanding of adolescent obsessive-compulsive personality traits and obsessive-compulsive symptoms from growth trajectories of perfectionism. Development and Psychopathology, 1-9. http://doi.org/10. 1017/S0954579421000195
Cite this article: Sametoğlu S, Denissen JJA, De Clercq B, De Caluwé E (2022). Towards a better understanding of adolescent obsessive-compulsive personality traits and obsessive-compulsive symptoms from growth trajectories of perfectionism ERRATUM. Development and Psychopathology 34: 1202, https://doi.org/10.1017/ S0954579421000547 